\title{
Construyendo una economía ecológica radical para la autonomía local
}

\author{
David Barkin \\ Universidad Autónoma Metropolitana, Ciudad de México, México \\ Email: barkin@correo.xoc.uam.mx \\ María Fernanda Ortega Valdez \\ Universidad Autónoma Metropolitana, Ciudad de México, México \\ Email: ferort.val@gmail.com \\ Marien Saldaña Guillen \\ Universidad Autónoma Metropolitana, Ciudad de México, México \\ Email: marien.uam@gmail.com \\ Cesar Mirafuentes de la Rosa \\ Universidad Autónoma Metropolitana, Ciudad de México, México \\ Email: cesar.mirafuentesuamx@gmail.com

\section{Tania Valentina Pérez-Riaño Arredondo} \\ Instituto Tecnológico de Oaxaca, Oaxaca, México \\ Email: valentina.priano@gmail.com
}

Resumen: La autonomía es el anhelo de millones de personas viviendo en comunidades alrededor del mundo. La Economía Ecológica Radical (EER) ofrece una metodología para entender y acompañarlas en sus luchas, consolidando sus sociedades, mejorando su calidad de vida y conservando sus ecosistemas. Incorporando conceptos medulares como: instituciones comunitarias, gobernanza y autogestión y redes de interacción y organización, examinamos múltiples casos de éxito de grupos que proponen mantenerse al margen de organización capitalista en las naciones de que son parte. Ofrecemos una necesaria y nueva interpretación teórica de la praxis de las organizaciones comunitarias y sus claras ventajas en la forma de relacionarse con la naturaleza.

Palabras clave: Ecología; comunidad; poscapitalismo; territorio; economía política

\section{Building a Radical Ecological Economy for Local Autonomy}

\begin{abstract}
Autonomy is the desire of millions of people living in communities around the world. Radical Ecological Economics (REE) offers a methodology to understand and support them in their struggles, consolidating their societies, improving their quality of life and conserving their ecosystems. Incorporating core concepts such as: community institutions, governance and self-management, and networks of interaction and organization, we examine multiple success stories of groups that propose to remain on the margins of capitalist organization in the nations of which they are part. We offer a necessary and new theoretical interpretation of the practice of community organizations and their clear advantages in the way they relate to nature.
\end{abstract}

Keywords: Ecology; community; postcapitalism; territory; political economy 


\section{Construindo uma economia ecológica radical para a autonomia local}

Resumo: A autonomia e o desejo de milhares de pessoas que vivem em comunidades ao redor do mundo. A Economia Ecológica Radical (EER) oferece uma metodologia para entender e acompanhar suas lutas, consolidando suas sociedades, melhorando sua qualidade de vida e conservando seus ecossistemas. Incorporando conselhos fundamentais como: instituições comunitárias, governança e autogestão e redes de interação e organização, examinamos várias histórias de sucesso de grupos que procuram se manter à margem da organização capitalista nos países dos quis fazem parte. Oferecemos uma necessária e nova interpretação teórica da prática das organizações comunitárias e suas claras vantagens na forma de se relacionar com a natureza.

Palavras-chave: Ecologia; comunidade; pós-capitalismo; território; economia política

$$
* * *
$$

\section{Introducción}

Los problemas medio ambientales a nivel mundial han incrementado el interés de la comunidad académica en torno a las condiciones de la naturaleza, generando nuevos enfoques teóricos tanto en las ciencias sociales como en las ciencias físicas y naturales. Algunas de estas propuestas facilitan nuestra capacidad de entender y colaborar con las comunidades organizándose para frenar las amenazas que atentan contra los ecosistemas y la vida de todas las especies. Uno de estos enfoques es la Economía Ecológica Radical (EER), inspirada en principios metodológicos y teóricos expuestos en la Economía Ecológica que retoma como uno de sus planteamientos primordiales los sistemas de conocimiento tradicional. La EER es una propuesta específica para examinar la transformación de la sociedad ante la devastación ecológica que ha ocasionado el modelo de mercado capitalista. La EER se desprende de muchos elementos del andamiaje teórico de las ciencias sociales heterodoxas, dado que es un enfoque que busca analizar los procesos de resistencia de las comunidades, y mostrar alternativas de organización ajenas al sistema capitalista, las cuales se hacen evidentes a través de sociedades poscapitalistas.

Esta propuesta de una EER da voz a aquellos pueblos que siguen luchando y resistiendo a las transformaciones violentas y destructivas que impusieron los sistemas colonial y capitalista. Con este marco analítico, se incorporan elementos centrales de las comunidades y sus cosmovisiones, para entender cómo sus alternativas de organización sociopolítica, económica y ecológica están contribuyendo a forjar otros mundos mejor equipados para enfrentar las crisis globales y locales.

El trabajo se organiza de la siguiente forma: la primera parte ofrece un mapeo de los elementos teóricos y metodológicos derivados de la formulación original de la EER (Barkin et al, 2012); en la segunda parte se propone incorporar nuevos elementos con base en la importancia de la construcción de instituciones comunitarias, el fortalecimiento de su autonomía, los procesos de gobernanza de los pueblos campesinos e indígenas y la asociación entre comunidades a partir de sus organizaciones; y, finalmente, en la tercera parte se propone una reflexión sobre los posibles caminos de discusión de la EER.

\section{Una introducción metodológica}

La EER es un proyecto analítico en construcción que propone explicar y potenciar la transformación de comunidades campesinas e indígenas. La edificación de conocimiento desde este enfoque es un reto epistemológico y ontológico. La Economía Ecológica, dentro en todos sus enfoques (Barkin et. al, 2012), plantea entender los procesos sociales-naturales-económicos como una unidad a través de diversos enfoques y metodologías: análisis multicriterio, transdisciplinariedad, multidisciplinariedad y pluralismo metodológico. Sin embargo, la EER considera fundamental la praxis social, los saberes tradicionales, el diálogo de saberes (Leff, 2003), la memoria biocultural (Toledo, 2008), el metaparadigma (Burkett, 2008) y la ciencia posnormal (Funtowicz y Ravetz, 2000) como marcos para estudiar la realidad de los pueblos campesinos e indígenas. Se busca superar la ciencia normal (Kuhn, 1962) que construyó un paradigma para la comunidad científica (Contreras, 2004). Por ello, desde la EER es necesario sustituir el paradigma dominante con enfoques alternativos de generación de conocimiento, enfatizando la inconmensurabilidad del patrimonio natural en términos económicos (Fuente, 
2008; Funtowicz y Ravetz, 2000). La EER cuestiona los límites epistemológicos de la ciencia normal, proponiendo otra forma de hacer ciencia basada en el reconocimiento de diversos actores dentro y fuera de las comunidades que contribuyen a la construcción de conocimientos a partir de la praxis.

Este marco pretende trascender el carácter científico normativo que las teorías ortodoxas imponen al análisis de los fenómenos económicos, sociales y naturales. Se reconoce una diversidad de pensamientos, cosmovisiones, disciplinas y teorías necesarias para estudiar conflictos ecológicos, posiciones comunitarias políticas, procesos productivos, distributivos, naturales, culturales e ideológicos en los que los sujetos de la comunidad están inmersos.

La EER incorpora categorías marxistas para tratar de explicar el contexto en el que las comunidades campesinas e indígenas se enfrentan al sistema capitalista bajo su dinámica de acumulación del capital que es producto de: la subsunción formal y real de la fuerza de trabajo y su enajenación a través de una lucha de clases, que genera excedentes mediante la producción de mercancías y forja al sujeto revolucionario. La categoría del excedente es central en el enfoque marxista. Es la parte del producto social generada por los trabajadores y apropiada por los capitalistas. En términos físicos este excedente es la parte de la producción que va más allá de lo necesario socialmente para la reproducción de los trabajadores. El excedente enuncia una relación social en la que se definen las clases sociales: los productores y los que se apropian el excedente. Baran (1958) dilucidó y criticó el concepto, aseverando que depende del estado de la lucha de clases sociales. Sugirió que hay un excedente real y potencial; la parte del producto social que se invierte y se acumula para la reproducción de las clases sociales (que no son proletarios) y la «discrepancia que existe entre la renta nacional alcanzable con el uso pleno del stock de capital productivo y la fuerza de trabajo existente y la renta nacional realmente conseguida» (Arriola, 1989, p. 357); es decir, el excedente potencial es aquella parte del producto que el sistema no es capaz de aprovechar.

Según Baran, su aportación contribuye a explicar el subdesarrollo, mostrando cómo el capitalismo monopolista impide utilizar el excedente para fines sociales e inmoviliza las capacidades que no le son útiles (Baran y Sweezy, 1968). El excedente no es particular del sistema capitalista, ya que existe en todas las sociedades y su función cambia en cada una de ellas. Es una categoría que históricamente caracteriza las formas de organización económico-sociales de cualquier sociedad. Particularmente, en el caso de comunidades campesinas e indígenas, no se limita a la esfera económica, y mucho menos en términos capitalistas con un carácter de subdesarrollo o tercermundista y bajo una relación de explotación del trabajo. En las comunidades, el excedente tiene formas tangibles e intangibles dentro de su producto social total, necesarios para su reproducción social. Los elementos tangibles podrían contribuir a su bienestar mediante la producción y la distribución colectiva de sus recursos. Los elementos intangibles son aquellos procesos culturales, políticos e ideológicos que facilitan su organización y su cohesión, es decir sus creencias, instituciones, y formas de llevar la vida (Sánchez, 2020).

Otra categoría fundamental, el sujeto revolucionario; también ofrece diferentes interpretaciones. En el Manifiesto del Partido Comunista se explica que uno de los fundamentos del modo de producción capitalista es la relación antagónica entre la clase trabajadora y la clase capitalista. La clase trabajadora es desposeída de los medios de producción; para sobrevivir tiene que vender su fuerza de trabajo a cambio de un salario; la clase capitalista organiza la producción y el proceso del trabajo con el objetivo de acumular y valorizar su capital con el excedente. La ruptura de dicha relación antagónica implicaría una transformación del modo de producción, y bajo esta lectura los trabajadores productivos son los únicos que pueden emprender dicha transformación, a partir de su conciencia de clase. Dados los limitantes de la visión tradicional del sujeto transformador ${ }^{1}$, desde la EER se propone la idea de un sujeto revolucionario que va más allá de la homogeneidad de sujetos y acciones. Por ello, surge la necesidad de reconocer diversas formas de revolución y transformación. Particularmente proponemos al sujeto revolucionario comunitario (Barkin y Sánchez, 2019) determinado por sus capacidades sociales emancipadoras, tanto objetivas como subjetivas. La comunidad se organiza bajo procesos de producción y gestión del territorio de forma colectiva, y bajo una estructura de gobernanza del bien común y sustentabilidad.

Nicholas Georgescu-Roegen, considerado como el padre de la Economía Ecológica moderna, mostró el significado de la segunda ley de la termodinámica para el sistema económico (1971). Georgescu enfatiza que el 
sistema económico no puede ser ajeno a las leyes de la naturaleza. En el sistema capitalista la naturaleza es vista como un factor que se transforma en productos bajo las necesidades sociales de reproducción del capital, sin importar su agotamiento o destrucción. Su observación es: «la materia-energía se está degradando continuamente de una forma disponible a una no disponible» (Georgescu-Roegen, 1994, p. 192); es decir, este sistema económico transforma a la naturaleza de forma irreversible ${ }^{2}$, de manera que el orden natural se ha reconfigurado tras las modificaciones que implica la dinámica de acumulación del capital, generando entropía. El resultado de esta transformación es que la vida terrestre está por extinguirse, dadas las leyes naturales que gobiernan el planeta y en las que el sistema capitalista se relaciona y se sujeta.

Siguiendo con este análisis, James O’Connor planteó la segunda contradicción del capitalismo (2001, p. 195). Las condiciones de producción capitalista constituyen barreras al desarrollo producidas por el propio sistema, las cuales derivan en crisis. Dentro de dichas barreras, la contaminación y destrucción de la naturaleza es una de ellas, la cual no es externa y surge de la misma naturaleza del capital, como consecuencia de los procesos productivos destructivos capitalistas.

Otro elemento importante es el concepto de metabolismo social al estudio de la relación entre los procesos económicos y la naturaleza (Foster, 2000; Altvater, 2006; Burkett, 2008; Toledo, 2013; Barkin, 2019). Este concepto surge originalmente de las aportaciones de Marx: «El trabajo es, independiente de todas las formaciones sociales, condición de la existencia humana, necesidad natural y eterna de mediar el metabolismo que se da entre el hombre y la naturaleza, y, por consiguiente, de mediar la vida humana» (Marx, 2008, p. 53). El metabolismo entre el hombre y la naturaleza está determinado por relaciones sociales específicas. En el caso del sistema capitalista, estas relaciones sociales explican una parte fundamental del metabolismo social entre la sociedad y la naturaleza; a través del proceso de trabajo ${ }^{3}$ y el proceso de producción de valores (dada la dualidad del trabajo, entre trabajo concreto y abstracto) (Altvater, 2006).

Hay diversos estudios empíricos donde el metabolismo social explica el impacto ecológico de los procesos productivos capitalistas en determinados lugares o regiones; reflejan los flujos de materia y energía, a la entrada y salida de estos procesos a nivel material o tangible. Toledo (2013) insiste que no es suficiente estudiar el metabolismo materialmente ${ }^{4}$; además, es fundamental entender los procesos intangibles en el metabolismo, basados de elementos simbólicos, leyes, normas, creencias, cosmovisiones, signos, imaginaciones, etc. Son necesarios para explicar la organización social a través de instituciones y mercados.

Karl Polanyi (2007) por su parte, argumenta que históricamente, los sistemas económicos habían estado acompañados de los mercados de forma secundaria. Sin embargo, dentro del sistema capitalista se transforma la sociedad en una institución subordinada al mercado autorregulado ${ }^{5}$. Esto es particular del sistema capitalista: los mercados dominan todas las esferas de reproducción social, en donde la naturaleza, el trabajo y el dinero se convierten en mercancías ficticias, organizados y distribuidos según los designios del capital, es decir, la clase dominante. Polanyi tilda a estas mercancías como ficticias por no haberse producidas para su venta, ya que tienen determinaciones biológicas, culturales y políticas. La organización social basada en estas mercancías ficticias tiende al colapso total. En contraste con el paradigma dominante, Polanyi sostiene que la economía está arraigada a la sociedad (y al planeta) para su funcionamiento.

Finalmente, Iván Illich, un pensador utópico, anarquista, y crítico de la modernidad, postula que no son eternos el crecimiento económico y el bienestar social capitalista. Considera como límites las instituciones que sostienen el sector industrial (económico) y político ${ }^{6}$. Propone alternativas de organización social basadas en la convivialidad. Esta alternativa «descansará sobre contratos sociales que garanticen en cada uno el mayor y más libre acceso a las herramientas de la comunidad, con la condición de no lesionar una igual libertad de acceso al otro» (1978, p. 8) y en donde se garantice la organización propia de la sociedad, a través del trabajo y la defensa de la vida (Barkin, 2019a).

El concepto de convivialidad es aquella forma de vivir en comunidad bajo una organización colectiva de los medios de trabajo y modos de producción. Illich, considera que las comunidades organizadas y armonizadas en diferentes aspectos, viven bajo el carácter austero que «no implica aislamiento, o reclusión; sino lo que funda la amistad; sería una virtud que sólo excluye los placeres que degradan la relación personal» (Esteva, 2012, p. 8). 
La austeridad comunitaria y convivial reconoce los límites -físicos, biológicos, ecológicos, culturales, económicos- de los elementos que los sujetos ocupan para reproducirse.

\section{Las instituciones: Autonomía sin Aislamiento}

En todo sistema social existen instituciones, es decir, «un conjunto de roles definidos por pautas de valor que orientan la acción de cada uno de sus miembros» (Parsons, 1999). Las instituciones determinan las formas de interacción social elementales, que van desde la organización de la producción hasta el establecimiento de las relaciones de poder. Cada miembro de un sistema social posee un estatus, o en otros términos una posición social, que está determinado por un rol particular establecido por una institución. Es mediante la interacción social que el estatus y el rol se conjugan para adquirir un significado social propio de una institución. Las pautas de valor que configuran los roles se construyen a través de la cosmovisión y la cultura de cada sociedad. De tal suerte que, una institución integra elementos motivacionales y culturales o simbólicos. Sin embargo, las instituciones cumplen un papel que va más allá de la determinación de roles y estatus, son un mecanismo de socialización a través del cual se crean los vínculos primarios, esenciales para lograr la vida en comunidad.

La idea de que los seres humanos poseen una individualidad congénita dada por cuestiones biológicas o naturales resulta equivocada y responde a mecanismos ideológicos impuestos por el capital. En contraste, se considera que su existencia, a lo largo de la historia, ha dependido de la interacción social, en concreto, de la acción colectiva y de su relación con otras especies, el medio ambiente y la naturaleza. Entonces, ¿¿cómo es posible explicar los procesos históricos que han articulado la idea de un ser humano atomizado en busca de su plena individualidad para autorrealizarse? La respuesta a esta pregunta se encuentra en la degradación de los vínculos primarios por parte del capital a través de la instauración de sus instituciones donde domina su poder económico. La autoconservación de los seres humanos depende de la satisfacción de sus necesidades fisiológicas, impuestas biológicamente, pero los satisfactores y las formas en la que se obtienen carecen de un argumento biológico o natural, por lo cual es incongruente la idea de naturaleza humana (Fromm, 2008). Es el ser humano quien la construye a través de los procesos de socialización que se entrañan en las instituciones. En este sentido, las instituciones capitalistas han construido una naturaleza humana individualista, que además de ser artificial, e incluso antinatural, es imposible de demostrar ${ }^{7}$.

El ser humano ha recurrido a la vida en comunidad por una necesidad que es igual de fuerte al impulso biológico de saciar su hambre para no morir de inanición, puesto que, de vivir aislado sufriría de una autodestrucción mental que lo llevaría a la muerte con seguridad. De esta forma, entre mayor es el grado de independencia y libertad que tiene el ser humano, para constituirse como individuo, el miedo al aislamiento y a la incertidumbre de hacerle frente al futuro crecen:

«... el hombre, cuanto más gana libertad, en el sentido de su emergencia de la primitiva unidad indistinta con los demás y la naturaleza, y cuanto más se transforma en «individuo», tanto más se ve en la disyuntiva de unirse al mundo en la espontaneidad del amor y del trabajo creador o bien buscar alguna forma de seguridad que acuda a vínculos tales que destruirán su libertad e integridad de su yo individual. ... El hombre le teme a la soledad, a enfrentarse él mismo a situaciones futuras adversas, a la incertidumbre que crea el futuro. En la comunidad, el hombre encuentra refugio, se hace a sí mismo como un ser libre para alcanzar la plenitud del yo, no es libre para liberarse de... [...]» (Fromm, 2008, p. 59).

En la comunidad, el ser humano se reconforta a sí mismo ante los peligros que le acechan en el individualismo; la comunidad le dota de pertenencia y sentido de orientación. La comunidad se consolida a través de la relación de sus miembros con su entorno y su contexto social, a saber, en la internalización de los vínculos primarios, en donde sus miembros aprenden a coexistir con la naturaleza. Bajo este contexto, la comunidad otorga a sus miembros libertad para la autorrealización, contraria a la libertad de autorrealización, esta última basada en el individualismo metodológico. La degeneración de los vínculos primarios proviene de los procesos de socialización que las instituciones industriales han imputado a la sociedad, los cuales han corroído el sentido de las relaciones del ser humano con otras especies, el medio ambiente y la naturaleza. 
Las instituciones industriales (Illich, 1978) han invertido el sentido de las máquinas. Se creía que las herramientas que el ser humano utiliza para realizar sus actividades productivas estarían al servicio y bajo control de la humanidad, lo cual ha sido contrario desde el afianzamiento de las sociedades industriales. En su evolución, el capital creó instituciones industriales que le permitieran reproducirse o, en otros términos, desplazó a aquellas instituciones que se lo impedían, desvirtuando así los vínculos primarios de la sociedad. Son estas instituciones las que han creado una disonancia con la naturaleza, pues han provocado una relación de existencia contraria a la de coexistencia.

Con relación al ámbito económico, las instituciones, además de determinar la organización de la producción, modelan las relaciones de intercambio, consumo y distribución (Veblen, 1963; Baudrillard, 1974). Es por ello por lo que el análisis de las instituciones ofrece una comprensión robusta acerca del modo de producción en las comunidades que se definen como sociedades poscapitalistas ${ }^{8}$. En este sentido, la EER da a las instituciones un papel importante para explicar por qué algunas comunidades funcionan bajo una organización distinta a la capitalista. Enriquecida por otras disciplinas como la etnoecología, la EER identifica aquellas instituciones que son claves para el desarrollo y la consolidación de las sociedades poscapitalistas; facilita distinguir aspectos relevantes de las instituciones que relaciona la sociedad con la naturaleza.

La etnoecología identifica los conocimientos ambientales que provienen de una tradición cultural específica (Milton, 1997), apelando al relativismo cultural y al particularismo histórico. Mediante el bricolage (hacerlo uno mismo), estructura diversos fragmentos de una comunidad (recursos arqueológicos, históricos, antropológicos y empíricos) (Lévi-Strauss, 1964) para reconocer los procesos de generación y transmisión de conocimientos ambientales de las instituciones, que socializan entre sus miembros, los cuales son fundamentales para la comprensión de los procesos económicos dentro de las sociedades poscapitalistas; encontramos muy productivo realizar estos trabajos en colaboración con los propios miembros de la comunidad (Fuente, et al., 2018). Los estudios etnoecológicos se caracterizan por ser integrales, ya que analizan los sistemas de creencias y cosmovisiones, los conocimientos ecológicos tradicionales, las prácticas productivas, y las instituciones locales para el manejo y utilización del patrimonio natural (Ruiz Mallén, et al., 2012). La aplicación de la etnobiología también puede ser de gran utilidad para identificar los ciclos biológicos que han sido establecidos por las instituciones de cada comunidad (de Albuquerque, 1999).

En su búsqueda de la comprensión y del empoderamiento de las comunidades, la EER ha incorporado el concepto de la memoria biocultural, reuniendo un conjunto de procesos biológicos humanos y procesos culturales, para acercarse a las prácticas ecológicas comunitarias. Ambos se co-determinan en la praxis: la diversidad biológica, los paisajes, la identidad, la lingüística, influyendo en la reproducción social y la natural. Como parte de lo mismo, se ha buscado definir el patrimonio biocultural, una categoría que explora la relación del hombre con la naturaleza (Luque, et al., 2018; Boege, 2008). Es decir, se parte del hecho de que a partir de la interacción con la naturaleza los seres humanos construyen patrones culturales que determinan su cosmovisión y lenguaje, el territorio, el conocimiento y las prácticas culturales aceptadas. El patrimonio biocultural va más allá de los recursos naturales con los que cuenta una comunidad. En la continuidad de su influencia cultural, cada paisaje, cada especie y cada objeto que pertenece a la naturaleza y forma parte de la cultura de una comunidad le dota de sentido e identidad; en este concepto se conjuga lo sagrado, lo ritual y lo espiritual; por esa razón, cultura y naturaleza es un binomio que se concreta en el patrimonio biocultural, imprescindible para los estudios de la EER. Mediante su comprensión se identifican aquellos elementos de la naturaleza con los cuales las comunidades se sienten fuertemente arraigadas para su protección y conservación, y la comprensión que se tiene del ciclo biológico de los recursos naturales, lo cual es fundamental en la gestión comunitaria y los procesos de autonomía para la libre autodeterminación.

Finalmente, el concepto de autonomía implica el rechazo de dominación que una clase tiene sobre otra, y distingue entre poder sobre y poder hacer, en donde el primero radica en la subordinación de una clase sobre otra y el segundo es la capacidad que tienen los subordinados de salir del proceso de dominación, de emanciparse. La autonomía rechaza el poder «sobre», característico del capitalismo; es decir se aleja de la concepción de dominación sobre los otros. Las instituciones son pues, el medio que permite generar la autonomía como un mecanismo de libre ejercicio determinación en todo aspecto y sentido. 


\section{Gobernanza y Autogestión}

El enfoque prevaleciente de las estructuras sociales se encuentra fundamentado en aquellas propuestas que dan fuerza al modelo hegemónico, donde las instituciones tienen un fuerte arraigo en la sociedad contractual; es decir, la acción colectiva se da en un contexto donde el individuo racional sólo busca organizarse con el fin único de maximizar su propio beneficio, y la sociedad colectivizada bajo el contrato social es resultado de las decisiones individuales (Villoro, 2003). Dentro de esta lógica, cabría preguntarse: ¿existe alguna alternativa institucional donde un grupo de individuos puedan organizarse para conseguir un bien común o colectivo?

Hay antecedentes de alternativas epistemológicas que sugieren que la vida colectiva se trata de una relación intersubjetiva con el territorio y patrimonios naturales. A partir de la cosmovisión que coexiste entre lo sagrado y lo cotidiano del ser en las comunidades se dan o mantienen procesos de interacción con el entorno y los seres que lo habitan -incluidas las personas- de manera situada o territorial. El espacio ${ }^{9}$ apropiado es considerado un factor de continuidad y de estabilidad para la identidad y la cohesión del grupo o colectividad (Esteban, 2012; Mazabel, et al., 2016).

Estas otras formas de coexistir en el mundo gestan alternativas que se dirigen por el interés del todo. «La comunidad se distingue de una sociedad por contrato. Esta última es resultado de las decisiones individuales de los contratantes dirigidos por particulares. La comunidad, en cambio se dirige por el interés del todo» ${ }^{10}$ (Villoro, 2003, p. 25).

Son sociedades campesinas e indígenas que pueden pensarse como: «nodos de relaciones sociales; como una trama de vínculos donde individuos y familias se adscriben a un sentido identitario de comunidad que les permite representarse y actuar en conjunto como una entidad social distinta y distinguible del exterior» (Garibay, 2008, p. 38). Son comunidades donde se gestan otras realidades y que se autodeterminan a partir de la cohesión social, el trabajo comunitario, la centralidad de territorio, la autonomía, la autosuficiencia, la formación político cultural, las redes de apoyo, la diversificación productiva y de mercado, y la gestión sustentable de recursos regionales (Barkin, 2018).

Bajo la praxis de las comunidades, se estudian diversas formas de interacción con la naturaleza, en donde se internalizan las instituciones y diversos procesos productivos, políticos e ideológicos. Para llevar a cabo la organización comunitaria es indispensable la participación de los sujetos en la construcción de la gobernanza desde abajo; esta gobernanza es compuesta por autonomía y autodeterminación; tanto política y territorial como educativa. Es la base de una transición a la democracia directa y la construcción de una identidad indígenacampesina (Fuente, et al., 2019).

La autodeterminación política es resultado del entramado que se construye gracias a la cohesión social de la comunidad, mediante la participación de todos los representantes (mujeres y hombres) de cada familia en la asamblea que es la máxima autoridad en la comunidad, dirigida por autoridades fincadas en el prestigio, en donde el trabajo es el resultado de la capacidad de coordinación y obediencia, a través de la acción colectiva. La representación política en una comunidad es el resultado de una convivencia directa y diaria; refleja el conocimiento profundo de cada ciudadano (prestigio), bajo el principio de la equidad intergeneracional, pues el ciudadano o ciudadana demuestra sus capacidades desde que es infante; ya en edad avanzada habiendo demostrado su entrega y aptitudes que es tomado o tomada en cuenta para dirigir u orientar el devenir de la comunidad (Martínez, 2009).

En el contexto de la autonomía política, las acciones llevadas a cabo por comunidades campesinas e indígenas se encuentran en disputa con las actividades llevadas por el Estado capitalista. La búsqueda de mantener una autonomía dentro de los procesos sociales de estos pueblos va desde la superación de injusticias hasta la construcción de instituciones, y formas alternativas tradicionales de tomar decisiones. A nivel interno, la autonomía política requiere de autodeterminación de actividades propias de la comunidad y a nivel externo, supone la creación y/o fortalecimiento de alianza con otras comunidades. Esta autonomía indígena va más allá de los límites y el poder del Estado que instaura derechos y obligaciones encasillados a un solo contexto, alejado de otros lenguajes, de otras formas de vida y de otras formas de valoración más allá de lo económico 
(Maldonado, 2009). La negación y la falta de visibilidad global de diversas formas de transformación social desarrolladas en las comunidades indígenas y campesinas, ha llevado a la construcción de autonomías políticas comunitarias en organizaciones antagónicas al sistema capitalista.

La autodeterminación territorial tiene implicaciones complejas que no se incorporan fácilmente a los conceptos imperantes del significado de tierra o propiedad basados en el mercado (Barkin y Lemus, 2015). Por ejemplo, en México, los bosques son sistemas naturales en espacios compartidos por distintos apropiadores; sin embargo, también tienen la característica de ser el hábitat necesario para la vida colectiva de comunidades, sus actividades, autogobierno y reproducción cultural y social. Sería importante establecer que el territorio es parte de la esencia social de las comunidades; lo que se configura, en principio, porque se gesta al interior de cada comunidad, prevalece el uso familiar de la propiedad, la tenencia de carácter tradicional; elementos de la esencia que cada comunidad respeta con todas las de la ley, una ley comunitaria, que se reproduce porque la propiedad pertenece a la comunidad y puede usarse en términos familiares.

Esta autodeterminación territorial debe estar acompañada de una serie de acciones que aseguren la capacidad social de auto-gobierno y la solidaridad del grupo. Además, para garantizar la consolidación de este proceso, también es importante la implementación de actividades capaces de generar excedentes para su uso en el mejoramiento de la calidad de vida y el cuidado de sus entornos naturales. Los excedentes materiales pueden ser reforzados con la movilización de diversas capacidades sociales y de recursos intangibles disponibles para la organización social y productiva de la comunidad, donde los procesos que desarrollan ${ }^{11}$ dentro de sus sistemas productivos, les permiten asegurar su capacidad de mantener y fortalecer su comunidad, satisfacer sus necesidades básicas y facilitar el intercambio con sus pares en el mercado como un ejercicio de su autodeterminación económica (Barkin, 2017; Barkin y Lemus, 2015). Dado que el territorio es fundamental para la reproducción de las comunidades, es importante recalcar que la autonomía territorial es fundamental para plasmar la identidad de los sujetos que se reivindican a partir de su trabajo colectivo y el goce en el territorio. La autonomía territorial es una forma de construir sociedades desde fuera del sistema capitalista, ya que el territorio para las comunidades representa la lucha y la resistencia a través de la autogestión territorial.

Estas dinámicas en las que las comunidades han interiorizado la necesidad de transformarse y de las cuales emergen formas de autodeterminación que permiten la cohesión y fortalecimiento de su gobernanza y autogestión, son antecedentes de cómo la praxis proporciona un nuevo sentido a la noción del «diálogo de saberes», hacia la reivindicación de la práctica cotidiana de las diferentes comunidades. Para la EER, esto es uno de los puntos de partida para crear mecanismos que construyan el deseo que muchos pueblos tienen de forjar una economía social y solidaria, capaz de satisfacer las necesidades de la comunidad sin menoscabo de los recursos naturales (Barkin, 2017).

\section{Red de organización y asociaciones nacionales e internacionales}

Es común que, desde las sociedades capitalistas, los grupos étnicos y comunidades indígenas o campesinas sean considerados como formas primitivas, que carecen de organización y estructuras sociales complejas. La concepción de estructuras sociales distintas a las sociedades que se pueden observar en el entorno urbano y bajo un contexto estrictamente capitalista, con frecuencia resulta difícil para las sociedades occidentales. Se debe comprender que, las estructuras sociales que se desenvuelven dentro de capitalismo se caracterizan por arraigar un funcionamiento social, económico y político que va hacia una sola dirección, «el progreso». Entendido en este sistema como la panacea o solución a los problemas económicos y ambientales, así como, el factor que contribuye a la acumulación. Lo anterior bajo la firme creencia de que el progreso y el crecimiento económico forjan las bases que conducen a la mitigación de problemas de desigualdad y problemas ambientales.

Ante el contexto mencionado, se concibe la necesidad de modernizar, para avanzar. Domina la idea ilusoria de que los cambios tecnológicos, las innovaciones y los megaproyectos llevarán a la mejora en las condiciones de vida en sociedades que carecen de estas capacidades. Bajo esta misma forma de pensamiento es que se entreteje la falsa concepción sobre sociedades/comunidades, que viven en el atraso social, tecnológico y económico. 
La EER da cuenta de muchas sociedades que se están construyendo al margen del sistema capitalista, que dan muestra de estructuras que no solo han sido malinterpretadas desde occidente, sino que, además, han sido subestimadas económica y políticamente. Son sociedades que, por lo general, han surgido en la periferia del mundo y específicamente en el Sur Global. Enfrentan el patrón teórico en el que se fundamenta el sistema y que promueve condiciones para la acumulación de capital y la restauración del poder de clase y que lo logra mediante la privatización, la financiarización, la administración y la manipulación de crisis y las redistribuciones estatales (Harvey, 2004).

Se trata, en la mayoría de los casos, de sociedades cuya configuración política se caracteriza por un nivel de autonomía interesante y una capacidad de gestión colectiva que se ve reflejada en la capacidad productiva y en la capacidad de cobertura de sus necesidades y requerimientos de vida básicos. Por lo general, sus estructuras se fortalecen cuando tienen como eje central y común, la lucha por el control colectivo de los recursos, por el derecho a la autodeterminación de sus modos de vida, contra las formas depredadoras sobre el despojo del territorio, agua, bosques, biodiversidad y conocimiento tradicional. «Estas sociedades u organizaciones no están involucradas en la simple formación de una economía alternativa, sino más bien una alternativa a la economía» (Barkin y Lemus, 2015, p. 40).

La fortaleza de estas estructuras no sería posible sin la interacción entre comunidades/sociedades a nivel local, regional e internacional. Esto se refleja en la conciencia y confianza que las mismas tienen en el nivel de integración. Entre algunos referentes empíricos internacionales se encuentran la Red de La Vía Campesina ${ }^{12}$, con una presencia en más de ochenta países. Por su parte, el Consorcio TICCA ${ }^{13}$, funge como una Asociación Civil Internacional con participación en 80 países y cuyos objetivos se sustentan en apoyo a los territorios que son conservados por pueblos originarios.

En América Latina, por mencionar algunos, se encuentra la resistencia de Movimientos de los Sin Tierra en Brasil que se ha basado en la reforma agraria como eje de lucha; no obstante, su conexión directa con la tierra los ha orientado a ir mucho más allá de la recuperación y sociabilización de tierras acaparadas para buscar nuevas formas de producción. En este mismo sentido, el Consejo Cívico de Organizaciones Populares e Indígenas de Honduras (COPINH); una organización social y política creada para la reivindicación y reconocimiento de los derechos políticos, sociales, culturales y económicos, impulsa el mejoramiento de las condiciones de vida de los pueblos y comunidades indígenas de Honduras. También, ha sido un espacio generador de debate y análisis constante de la coyuntura del país, que fomenta acciones y propuestas de manera permanente (Navarro y Pineda, 2009).

En México, un número considerable de organizaciones buscan el fortalecimiento de la autonomía política y la gestión adecuada de los sistemas naturales en los que habitan. Entre estos grupos destacan Otros mundos AC Chiapas ${ }^{14}$, con sede en San Cristóbal de las Casas, cuya principal actividad consiste en el desarrollo de estrategias de defensa del territorio y las organizaciones REMA (Red Mexicana de Afectados por la Minería) y ANAA (Asamblea Nacional de Afectados Ambientales) ${ }^{15}$.

A nivel internacional, lo que todas estas organizaciones tienen en común es, el fomento a las actividades de conservación ambiental y reproducción de los pueblos originarios y campesinos a través del ejercicio de la gobernanza y la organización política autónoma, teniendo como pilares la búsqueda de soberanía alimentaria, y la justicia social y ambiental. El impacto de este tipo de interacciones se ejemplifica muy bien con, la experiencia del movimiento mesoamericano que logró articularse en torno al eje de las represas a través de los Foros Mesoamericanos contras las Represas, al Movimiento Mesoamericano contra el Plan Puebla Panamá y a la Semana por la Diversidad Biológica y Cultural, cuyos procesos dieron inicio en el año 2001 en Chiapas, así como a la lucha centroamericana contra el Tratado de Libre Comercio Estados Unidos-Centroamérica (CAFTA) y al Movimiento Centroamericano contra las minas creado en 2005 (Castro, 2008).

Esto nos permite repensar la forma en la que estas otras sociedades funcionan tanto política como económicamente. De manera evidente, no se trata de comunidades que se encuentran en aislamiento o sin interacciones con su entorno geográfico inmediato y lejano; por el contrario, se trata de comunidades con plena conciencia de la necesidad de interactuar para fortalecer sus instituciones políticas, sociales y económicas. 
Estas interacciones no se reducen exclusivamente a organismos que apoyan sus lógicas y dinámicas como los ejemplos ya antes mencionados, sino que, además, se llevan a cabo con organismos gubernamentales, aunque tales interacciones son de carácter más selectivo y en función de las necesidades de comunicación, negociación y apoyos que la comunidad requiera.

«Las estrategias de las comunidades pueden ser exitosas si se logra asumir exitosamente el control sobre la gestión de sus recursos humanos, naturales y materiales mientras mejoran su capacidad de definir los límites de sus esfuerzos en autogobernanza, especificando las esferas de autonomía local y los contactos con los mercados nacionales e internacionales, a fin de impedir un inevitable movimiento autodestructivo hacia la autarquía» (Barkin, 2009, p. 317).

Las redes de interacción cumplen una función fundamental en la concreción y emancipación de las sociedades poscapitalistas. Evitan el aislamiento o la autarquía de estas otras sociedades sin que ello represente una pérdida de su identidad, su herencia cultural-simbólica y su autonomía política, institucional y organizativa.

\section{La EER como respuesta epistemológica a los desafíos ambientales}

Los desafíos ambientales contemporáneos han marcado el surgimiento de una cantidad considerable de posturas teóricas que se plantean como respuesta o solución a la grave crisis ambiental. No obstante, el grueso de estos planteamientos ofrece soluciones superficiales y poco fructíferas, pues se gestan desde la firme idea de que el sistema no es el problema de la crisis ambiental. Resulta evidente que sus propuestas no ofrecen cambios que vayan en contra del propio funcionamiento del sistema económico capitalista. Este es el caso de los planteamientos gestados desde la economía ortodoxa y algunos planteamientos desde la heterodoxia, incluyendo la economía ecológica. Propuestas como el pago por servicios ambientales, bonos de carbono, normatividades productivas ambientales, economía circular o ecotecnologías se muestran como soluciones que invitan a intensificar o mantener las estructuras y los ritmos productivos propios del sistema, generan la ilusión de una reducción en el impacto ambiental ocasionado por la actividad productiva.

Sin embargo, la EER surge como una respuesta necesaria y crítica ante el propio funcionamiento del sistema económico. Esta crítica va más allá del planteamiento de soluciones de contención; por un lado, se estudian los procesos sociales, económicos y naturales desde los aportes de distintas disciplinas, desde la filosofía política, la biología y la ecología política. Enfatiza la discusión e interacción entre estas disciplinas como una crítica a la modernidad, lo que plantea la necesidad de replanteamientos epistemológicos y ontológicos. Por otro lado, esta propuesta es desde abajo, a través de las praxis comunitarias campesinas e indígenas, ya que es ahí en donde se analizan los problemas ecológicos, la reproducción de la vida humana y no humana, así como la construcción de otras sociedades; basadas en la preservación del entorno natural, y en la lucha y resistencia contra las dinámicas depredadoras del sistema capitalista.

Como hemos plasmado aquí, los movimientos de campesinos e indígenas se constituyen con fundamento en sus modos de ver y construir la vida, sus valoraciones éticas y morales; conocimientos tradicionales, saberes ancestrales, cosmovisiones y cosmovivencias. Desde la teoría o academia nos plantean el estudio ontológico de un ser: liberado a partir de la colectividad, y no del individualismo, con conciencia ecológica, más no consumista. Es, además, un ser con una posición de lucha y resistencia, y no de enajenación hacia el capital, por lo que, para poder entenderlo, se requieren principios epistemológicos desde su entorno y actuar.

Por lo anterior, el estudio de estas comunidades desde sus fundamentos, y sobre todo a partir de una visión crítica y alternativa, implica un reto para el investigador. Se plantea que la observación de estas comunidades no puede ser exterior de la realidad del investigador, o vistas como un objeto de estudio únicamente académico. Desde la EER se propone el acompañamiento a los sujetos de acción comunitaria en la construcción de sus sociedades, basadas en estrategias sustentables para la reproducción de la vida, ideales que refuercen su identidad cultural y sus sistemas de conocimientos, así como la planificación de proyectos que permitan la reproducción material de las comunidades, a través de principios solidarios y ecológicos. 


\section{Conclusiones}

La EER plantea entender las estrategias de organización social de las comunidades que se consideran parte del sistema natural; un sistema que figura como el espacio en donde se mueven e interactúan los fenómenos de las esferas políticas, culturales y económicas. Bajo esta forma unitaria de entender diferentes procesos sociales y naturales, la EER se permite crear un marco conceptual, construido a partir de diversas disciplinas y metodologías, con el objeto de generar una propuesta que va más allá de la dimensión económica y académica que se direcciona al entendimiento y acompañamiento de las realidades comunitarias, donde no sólo existe un camino para construirse, sino que a partir de referentes empíricos latentes en el marco de las resistencias locales y regionales, emanan alternativas de organización social y económica que permiten vislumbrar espacios para el colectivo y el bien común.

La EER identifica 5 principios básicos que se encuentran interconectados y que caracterizan estas sociedades. El primero está en relación con el acompañamiento comunitario. La teoría y la realidad de las comunidades campesinas-indígenas no pretenden ser dos caminos diferentes; por el contrario, la teoría y la praxis convergen, y es a partir de esta interacción que la EER genera no solo instrumentos críticos, sino que va más allá, generando propuestas que deriven en el empoderamiento y acompañamiento a los sujetos comunitarios para la construcción y explicación de sus realidades.

Subsecuentemente, se encuentran sus conocimientos tradicionales y su extraordinaria flexibilidad por el diálogo de saberes. Es decir, la preservación, aplicación y reconfiguración de los conocimientos tradicionales permite a estas sociedades entretejer sus formas de gobernanza, sus instituciones y sus estrategias y prácticas de gestión productiva y ambiental a través del diálogo de saberes» construyendo convergencias entre los conocimientos científicos y tecnológicos y los conocimientos tradicionales, lo que implica un replanteamiento de los constructos ontológicos occidentales en relación a la importancia y validación de estos últimos.

Los dos factores anteriores se revelarán en las formas de autodeterminación comunitaria, las cuales permiten la cohesión necesaria para la capacidad de gobernanza y autogestión en la búsqueda del bien colectivo, procurando la protección del entorno natural, conduciendo a estas sociedades al siguiente factor que es, las alternativas y organización social. Esto encuentra sustento en las alternativas de organización social basadas en la convivialidad austera que Illich sugiere como, una propuesta latinoamericana de bienestar social, en oposición al desarrollo económico y el decrecimiento.

Los 4 factores anteriores serán la base para el fortalecimiento institucional y redes de interacción. Es decir, las estructuras institucionales erguidas dentro de la dinámica de las comunidades ya sean de facto o con el reconocimiento de instancias occidentales, que se ven fortalecidas con la existencia de las redes nacionales e internacionales. Hace varias décadas se examina la crisis estructural del capitalismo ocasionado por la serie de factores internos y que ya no pueden ser resueltos.

«Cuando aparecen crisis que ya no pueden ser resueltas en los términos propios de cada era, surge la necesidad histórica de una nueva era y se abre un parteaguas para pasar a ella. (...) Pero la naturaleza y características de la nueva era no están escritas en las estrellas» (Esteva, 2009, p. 38).

Ante esto, cabe proponer como alternativa a la organización social dominante capitalista; a las sociedades poscapitalistas. Estas muestran plena conciencia de ello, a través de los 5 principios que se interconectan dentro de sus configuraciones y estructuras sociales, mismas que dan cuenta de sus interpretaciones y reflexiones en torno a los retos ambientales presentes y futuros, reflexiones que superan de manera considerable las propuestas conservadoras ortodoxas y heterodoxas. Las sociedades poscapitalistas tienen el reto de determinar los límites de tales convergencias e interacciones para preservar sus cosmovisiones y estructuras políticas y sociales. En momentos en donde el sistema capitalista acontece su declive como consecuencia de sus contradicciones, se propone el sujeto revolucionario desde la comunidad y elementos no sólo para la transformación, sino también para la construcción de sociedades alternativas desde fuera del sistema (Barkin, 2019b). 


\section{Notas}

${ }^{1}$ Contemporáneamente, los trabajadores productivos van en descenso, mientras que los trabajadores en los sectores servicios e informales se incorporan a las economías, además de contar con un mayor número de desempleados lo que provoca una desarticulación y polarización de la clase trabajadora.

${ }^{2}$ Hay una relación «dialéctica» según Georgescu-Roegen entre el proceso económico y la naturaleza, al mismo tiempo que se transforma ella, el sistema económico también lo hace de forma irreversible.

${ }^{3}$ En el proceso de trabajo hay una transformación directa de la materia y la energía en valores de uso. En el sistema capitalista ha incrementado la producción de valores de uso necesitados por la sociedad más allá de la necesidad biológica. Dichas necesidades de la sociedad son las necesidades del capital; este dicta qué y cuánto se va a producir; qué se va a transformar y qué se va a apropiar. En este proceso, el trabajo y la naturaleza son fundamentales para la explicación de los efectos ecológicos de la producción de más valores de uso, ya que hay una entrada y salida tanto de materia como de energía, es decir, encontramos la producción de valores de uso, por un lado, pero del otro lado, está la salida de desechos. En este proceso, el trabajo necesita de la naturaleza y la naturaleza del trabajo para su transformación en valores de uso específicos del sistema capitalista.

${ }^{4}$ El análisis material del metabolismo social considera cinco procesos: la apropiación, la transformación, la circulación, el consumo y la excreción.

${ }^{5}$ La autorregulación implica que la oferta es igual a la demanda a través de los precios, es decir, «toda la producción está destinada a la venta en el mercado y que todos los ingresos vienen de ella» (Polanyi, 2007, p. 124)

${ }^{6}$ Bajo instituciones económicas capitalistas se tiene fe al incremento de la productividad como vía de bienestar y en términos políticos, y desconocen las resistencias, luchas y alternativas.

${ }^{7}$ Los propios teóricos de la filosofía del liberalismo, que defienden la idea de un ser humano individual, han aceptado que la naturaleza de la cual parten es supuesta. Por ejemplo, Bobbio menciona que el iusnaturalismo, presupuesto filosófico pilar del liberalismo, se basa en una «concepción general e hipotética de la naturaleza del hombre, que prescinde de toda verificación empírica y de toda prueba histórica» (1989, p. 12).

${ }^{8}$ Barkin (2015) propone que una sociedad poscapitalista se conforma por sujetos que pertenecen a una comunidad indígena y/o campesina, los cuales han sido víctimas de la exclusión y violencia por parte del sistema colonial y capitalista, por lo que por decisión propia y bajo sus cosmovisiones construyen alternativas de organización económica y política, basadas en una conciencia colectiva y ecológica de los sujetos.

${ }^{9}$ El espacio es una formación social que surge a partir de factores simbólicos, de identidad y apego. La correlación de estos resulta en la apropiación del territorio como un proceso dialéctico por el cual se vinculan las personas y los espacios, dentro de un contexto sociocultural, desde los niveles individual, grupal y comunitario hasta el de la sociedad. Este proceso se desarrolla a través de dos vías complementarias, la acción transformación y la identificación simbólica (Moranta \& Pol Urrútia, 2005).

${ }^{10}$ Por lo tanto, se quiere aludir a que las alternativas rurales, campesinas o indígenas se diferencian por no hacer una exclusión al individuo, sino más bien, hacer una incorporación del territorio, naturaleza, familia, cultura, religión, él todo

${ }^{11}$ Estas sociedades están fortaleciendo sus actividades tradicionales y forjando nuevas estrategias que les permiten renovar sus conocimientos y métodos productivos ancestrales, incorporando elementos tecnológicos y avances científicos útiles, convirtiéndose en gestores e innovadores (Barkin, 2018).

${ }^{12}$ La Red de Vía Campesina, es un Movimiento Político Internacional, autónomo, que aglomera a millones de campesinos y pequeños productores, con el objeto de promover la soberanía alimentaria y la justicia social. https://viacampesina.org/es/

${ }^{13}$ Consorcio de Territorios y Áreas Conservados por Pueblos Indígenas y Comunidades Locales TICCA, es una Asociación Civil Internacional, con presencia en más de 80 países que tiene como objetivo, reconocer y legitimar la labor de los pueblos originarios en la conservación de sus territorios y áreas naturales, así como, generar estrategias que permitan defender la presencia de estas comunidades como formas de reproducción alternas y cuyo papel es fundamental en preservación de los entornos y áreas naturales en las que se localizan. https://www.iccaconsortium.org/index.php/es/

${ }^{14}$ Otros Mundos AC. de Chiapas, trabaja en defensa de los territorios y en contra de proyectos y megaproyectos que comprometan los sistemas naturales. https://otrosmundoschiapas.org/

${ }^{15}$ ANAA surgió como una respuesta organizativa popular contra el deterioro y la devastación ambiental dentro del país, cuyo objeto se sustenta en detener y reconstruir el deterioro ambiental a través de la lucha y la búsqueda del cambio en las políticas e instituciones. Busca hacer visibles los conflictos en torno al deterioro ambiental. La Red Mexicana de Afectados por la Minería (REMA) surge en 2008 en contra de la minería para fortalecer las luchas y movimientos locales de resistencia. El Movimiento Mexicano de Afectados por las Presas y en Defensa de los Ríos (MAPDER) aglutina frentes estatales, organizaciones sociales y civiles y a comunidades de todo México afectadas por la construcción de presas (Castro, 2008). http:// www.afectadosambientales.org/ 


\section{Bibliografía}

Altvater, E. (2006). ¿Existe un marxismo ecológico? En A. Borón, J. Amadeo y S. González (Coords.), La Teoría Marxista Hoy (pp.341-363). Buenos Aires, Argentina: CLACSO.

Arriola, J. (1989). Excedente potencial y desarrollo posible. Realidad, (10), 357-366. Doi doi:10.5377/ realidad.v0i10.5364

Baran, P. (1958). Sobre la evolución del excedente económico. El Trimestre Económico, 25 (10), 735-748. Recuperado de https://www.jstor.org/stable/20855461

y Sweezy, P. M. (1968). El capital monopolista. Ciudad de México, México: Siglo XXI.

Barkin, D. (2019). Social metabolism: a construct for teaching and research in ecological economics, En: D. Barkin y G. Carrillo (Eds.), Ecological Economics and Social-Ecological Movements Science, policy and challenges to global processes in a troubled world, (pp.47-56). Ciudad de México, México: Universidad Autónoma Metropolitana-Xochimilco.

(2019a). Conviviality. En: A. Kothari, A. Salleh, A. Escobar, F. Demaria y A. Acosta, (Eds.) Pluriverse: A Post-Development Dictionary (pp.136-139), New Delhi, India: Tulika Books.

y Sánchez, A. (2019b). The communitarian revolutionary subject: new forms of social transformation. Third World Quarterly, 1-23.Doi doi:10.1080/01436597.2019.1636370

(2018). La Promesa del Campesinado Mexicano. En: D. Barkin, De la protesta a la propuesta: 50 años imaginando y construyendo el futuro (pp.477-484). Ciudad de México, México: Siglo XXI.

(2017). La Economía Ecológica desde abajo. En A. Azamar, D. Escobar, y S. Peniche (Eds.), Perspectivas de la economía ecológica en el nuevo siglo, (pp.91-110). Guadalajara, México: Fondo Editorial Universitario, Universidad de Guadalajara, Sociedad Mesoamericana y del Caribe de Economía.

y Lemus. (2015). Construyendo mundos poscapitalistas. Cultura y representaciones sociales, 10 (19), 2660. Recuperado de http://www.scielo.org.mx/pdf/crs/v10n19/v10n19a2.pdf

Fuente, M. E., y Tagle, D. (2012). La significación de una Economía Ecológica radical. Revista Iberoamericana de Economía Ecológica, 19(1), 0001-0014. Recuperado de https://redibec.org/ojs/index.php/revibec/ article/view/194/84

(2009). Principios para la Construcción de Organizaciones Socio-Económicas Alternativas: Lecciones aprendidas desde el trabajo fuera de las Estructuras institucionales. Review of Radical Political Economics, 41 (3), 372-379. Doi doi:10.1177/0486613409336352

Baudrillard, J. (1974). Crítica de la economía política del signo. Ciudad de México, México: Siglo XXI.

Bobbio, N. (1989). Liberalismo y democracia. Ciudad de México, México: Fondo de Cultura Económica.

Burkett, P. (2008). La comprensión de los problemas ambientales actuales vistos con el enfoque marxista. Argumentos, 21 (56), 21-32. Recuperado de http://www.scielo.org.mx/scielo.php?pid=S0187-57952008000100002

Castro, G. (Junio de 2008). Nace la Red Mexicana de Afectados por la Minería (REMA). V Encuentro MAPDER y I Encuentro REMA. Defensa de los ríos (MAPDER) y el I Encuentro de la Red Mexicana de Afectados por la Minería (REMA) Temacapulin, México.

Contreras, R. (2004). El paradigma científico según Kuhn. Desarrollo de las ciencias: del conocimiento artesanal, hasta la ciencia normal. VI Escuela Venezolana para la Enseñanza de Química., 43-51. Recuperado de http:/ 
Polis, Revista Latinoamericana, $N^{\circ} 56 \mid 2020$

/webdelprofesor.ula.ve/ciencias/ricardo/PDF/Paradigma_Cientifico_segun_Kuhn.pdf

de Albuquerque, U. P. (1999). La importancia de los estudios etnobiológicos para establecimiento de estrategias de manejo y conservación en las florestas tropicales. Biotemas, 12 (1), 31-47. Recuperado de https:// periodicos.ufsc.br/index.php/biotemas/article/download/21903/19870

Esteban, J. M. (2012). Nuevo análisis de «La tragedia de los comunes». Teoría y praxis, 11, 40-58. Recuperado de https://dialnet.unirioja.es/descarga/articulo/4201668.pdf

Esteva, G. (2012). La convivialidad y los ámbitos de la comunidad: claves del nuevo mundo. Crítica de la cultura del progreso capitalista, 1-25. Recuperado de http://www.critica.org.mx/Esteva2.pdf

Foster, J. B. (2000). La ecología de Marx. Materialismo y naturaleza. Madrid, España: El Viejo Topo.

Fromm, E. (2008). La crisis del psicoanálisis en la vida contemporánea. Madrid, España: Paidós

Fuente, M. E. (2008). La economía ecológica: ¿Un paradigma para abordar la sustentabilidad? Argumentos, 21 (56), 75-99. Recuperado de http://www.scielo.org.mx/scielo.php?script=sci_arttext\&pid=S018757952008000100005

Fuente, M. E., Barkin, D., Esquivel, A.L. \& Ramos, M.F. (2018). La co-investigación en comunidades zapotecas de Oaxaca. Reflexiones hacia un diálogo intercultural. Sinéctica, 50. Recuperado de https://sinectica.iteso.mx/ index.php/SINECTICA/article/view/793

Fuente, M. E., Barkin, D., y Clark, R. (2019). Governance from below and environmental justice: Community water management from the perspective of social metabolism. Ecological Economics, 160, 52-61. Doi doi:10.1016/j.ecolecon.2019.01.022

Funtowicz, S., y Ravetz, J. (2000). La Ciencia Posnormal. Ciencia con la gente. Barcelona, España: Icaria.

Garibay Orozco, C. (2008). Comunalismos y liberalismos campesinos: identidad comunitaria, empresa social forestal y poder corporado en el México contemporáneo. Zamora, México: El Colegio de Michoacán.

Georgescu-Roegen, N. (1971). La ley de la entropía y el proceso económico. Lanzarote, España: Argentaria/Visor.

Georgescu-Roegen, N. (1994). ¿Qué puede enseñar a los economistas la termodinámica y la biología? En: F. Aguilera y V. Alcantara (Coords.) De la economía ambiental a la economía ecológica (pp.188-198). Barcelona, España: Icaria.

Harvey, D. (2004). The 'New`Imperialism: Accumulation by Dispossession. Socialist Register 2004: The New Imperial Challenge. 40, 63-87. Recuperado de https://socialistregister.com/index.php/srv/issue/view/441

Illich, I. (1978). La convivencialidad. Escuela Técnica Superior de Arquitectura de Madrid, España: Universidad Politécnica de Madrid: Ministerio de Vivienda.

Leff, E. (2003). Racionalidad ambiental y diálogo de saberes: sentidos y senderos de un futuro sustentable. Desenvolvimento e Meio Ambiente, 7, 13-40. Recuperado de http://ppgaa.propesp.ufpa.br/pdfs/prosel2020/ DAFDS/D3.pdf

Lévi-Strauss, C. (1964). El pensamiento salvaje. Ciudad de México, México: Fondo de Cultura Económica.

Luque, D., Martínez-Yrízar, A., López, G., y Murphy, A. D. (2018). Los complejos bioculturales. En: V. M. Toledo y P. Alarcón-Cháires (Eds.), Tópicos Bioculturales (pp.7-33). Morelia, México: Universidad Nacional Autónoma de México. 
Polis, Revista Latinoamericana, $N^{\circ} 56 \mid 2020$

Maldonado, B. (2000). Autonomía y Comunalidad India. Enfoques y propuestas desde Oaxaca. Oaxaca, México: Secretaría de Asuntos Indígenas del Gobierno del Estado/Coalición de Maestros y Promotores Indígenas de Oaxaca/Centro de Encuentros y Diálogos Interculturales.

Martínez, J. (2009). Eso que llaman comunalidad. Oaxaca, México: CONACULTA/Secretaría de Cultura, Gobierno de Oaxaca/Fundación Alfredo Harp Helú.

Marx, K. (2008). El Capital. Tomo I. Libro 1. Ciudad de México,México: Siglo XXI.

Mazabel, D. G., Miranda, G. A., y González-Fuente, Í. (2016). Identidad y Gestión Comunitaria del Territorio en un Ejido de Guanajuato,. Antropología Experimental, 16, 63-78. Doi doi: 10.17561/rae.v0i16.2895

Milton, K. (1997). Ecologies: anthropology, culture and the environment. International Social Science Journal, 49 (154), 477-495.Doi doi:10.1111/j.1468-2451.1997.tb00039.x

Moranta, V. T., y Pol, E. (2005). La apropiación del espacio: una propuesta teórica para comprender la vinculación entre las personas y los lugares. Anuario de Psicología, 36 (3), 281-297. Recuperado de http://diposit.ub.edu/ dspace/bitstream/2445/99095/1/545803.pdf

Navarro, M.L. y Pineda, C.E. (2009). Luchas socioambientales en América Latina y México: nuevas subjetividades y radicalidades en Movimiento. Bajo el Volcán, 14, 81-104. Recuperado de https://www.researchgate.net/ publication/237032120_Luchas_socioambientales_en_America_Latina_y_Mexico _Nuevas_subjetividades_y_radicalidades_en_movimiento

O’Connor, J. R. (2001). Causas naturales. Ensayos de marxismo ecológico. Ciudad de México, México: Siglo XXI.

Parsons, T. (1999). El sistema social. Ciudad de México, México: Alianza Editores.

Polanyi, K. (2007). La gran transformación: crítica del liberalismo económico. Ciudad de México, México: Fondo de Cultura Económica.

Ruiz, I., Domínguez, P., Calvet, L., Orta, M., y Reyes, V. (2012). Investigación aplicada en etnoecología: experiencias de campo. Revista de Antropología Iberoamericana, 7 (1), 9-32. Recuperado de https://www.redalyc.org/ articulo.oa?id=62322227002

Sánchez, A. (2020). Movilización del patrimonio comunitario, un mecanismo para la transformación Social y la construcción de sociedades post-capitalistas: El Totonacapan, Huehuetla, Puebla. (Tesis Doctoral). Universidad Autónoma Metropolitana, Ciudad de México, México.

Toledo, V. M. (2013). El metabolismo social: una nueva teoría socioecológica. Relaciones. Estudios de historia y sociedad, 34 (136), 41-71. Recuperado de http://www.revistarelaciones.com/index.php/relaciones/article/ view/163/192

Toledo, V. M., y Barrera, N. (2008). La memoria biocultural: la importancia ecológica de las sabidurías tradicionales. Barcelona, España: Icaria.

Veblen, T. (1963). Teoría de la clase ociosa. Ciudad de México, México: Fondo de Cultura Económica.

Villoro, L. (2003). De la libertad a la comunidad. Ciudad de México, México: Fondo de Cultura Económica/ Instituto Tecnológico y de Estudios Superiores de Monterrey. 